NASA Technical Memorandum 105686

AIAA-92-3082

\title{
A Fast, Uncoupled, Compressible, Two- Dimensional, Unsteady Boundary Layer Algorithm With Separation for Engine Inlets
}

Robert L. Roach and Chris Nelson

Georgia Institute of Technology

Atlanta, Georgia

Barbara Sakowski and Douglas Darling

Lewis Research Center

Cleveland, Ohio

and

Allan G. van de Wall

Case Western Reserve University

Cleveland, Ohio

Prepared for the

28th Joint Propulsion Conference and Exhibit

cosponsored by the AIAA, SAE, ASME, and ASEE

Nashville, Tennessee, July 6-8, 1992

\section{N/Sก}

$$
\begin{aligned}
& \text { (NASA-TM-105\$BS) A FAST, UNCOUPLFC, } \\
& \text { COMPKESSIOLE, TWO-DIMENSTONNAL, UNSTEADY } \\
& \text { GOUNGARY LAYER ALGOQITHM WITH SEPARATIOM: FOR } \\
& \text { ENGIAE INLETS (NAJA) Q D } \\
& \text { N92-27553 }
\end{aligned}
$$


$\mathrm{x}$ momentum

$$
\bar{\rho} \frac{\partial \bar{u}}{\partial \bar{t}}+\frac{\bar{\rho} \bar{u}}{R_{o}^{k}} \frac{\partial R_{o}^{k} \bar{u}}{\partial x}+\bar{\rho}-\frac{\partial \bar{u}}{\partial y}=\frac{\partial}{\partial y}\left(\frac{1}{R e} \frac{\partial \bar{u}}{\partial y}\right)-\frac{\partial \bar{P}}{\partial x}
$$

energy

$$
\begin{aligned}
& \bar{\rho} \frac{\partial \bar{T}}{\partial \bar{t}}+\frac{\bar{\rho} \bar{u}}{R_{0}^{k}} \frac{\partial R_{0}^{k} \bar{T}}{\partial x}+\bar{\rho} \bar{v} \frac{\partial \bar{T}}{\partial y}= \\
& \frac{\partial}{\partial y}\left(\frac{1}{\operatorname{RePr}} \frac{\partial \bar{T}}{\partial y}\right)-\frac{1}{\operatorname{Re}}\left(\frac{\partial \bar{u}}{\partial y}\right)^{2}+\frac{\partial \bar{P}}{\partial \bar{t}}+\bar{u} \frac{\partial \bar{P}}{\partial x}
\end{aligned}
$$

The variables were defined as:

$$
\begin{gathered}
\vec{\rho}=\frac{\rho}{\rho_{\max }} \\
\bar{u}=\frac{u}{u_{\max }} \\
\bar{P}=\frac{\rho}{\rho_{\max } u_{\max }^{2}} \\
\bar{T}=\frac{c_{p} T}{u_{\max }^{2}} \\
\bar{t}=t u_{\max } \\
R e=\frac{\rho_{\max } u_{\max }}{\mu_{\text {off }}}
\end{gathered}
$$

$R_{0}$ is the radius of the wall for axisymmetric flow $(k=1)$. For rectangular coordinates $R$, was ignored $(k=0)$. It should be noted that $\mathrm{Re}$ and time terms were not actually non-dimensional. $\operatorname{Re}$ had units of (length) ${ }^{-1}$. and time had units of length. Also, $x$ and $y$ (and $R_{h}$ ) remained dimensional. So. each term in each of the above boundary layer equations has units of (length) $)^{-1}$.

\section{SOLUTION ALGORITHM}

The momentum, continuity and energy equations were differenced as described by Kwon et al.[1]

For any scalar quantity $\phi$ :

$$
\left(\frac{\partial \phi}{\partial x}\right)_{i j}=\frac{\phi_{i j}-\phi_{i-1 j}}{x_{i}-x_{i-1}}
$$

$$
\left(\frac{\partial \phi}{\partial y}\right)_{i j}=\frac{\phi_{i j+1}-\phi_{i j-1}}{y_{j+1}-y_{j-1}}
$$

$$
\begin{gathered}
\left(\frac{\partial}{\partial y} \frac{1}{R e} \frac{\partial \phi}{\partial y}\right)_{i j}=\frac{\frac{1}{R e_{i j+\frac{1}{2}}} \frac{\phi_{i j+1}-\phi_{i j}}{y_{j+1}-y_{j}}}{\frac{1}{2}\left(y_{j+1}-y_{j-1}\right)} \\
-\frac{1}{R_{i j j} \frac{1}{2}} \frac{\phi_{i j}-\phi_{i j-1}}{y_{j}-y_{j-1}} \\
\left.\frac{\frac{1}{2}\left(y_{j+1}-y_{j-1}\right)}{\partial t}\right)_{i j}=\frac{\phi_{i j}-\phi_{i j}^{o l d}}{\Delta \bar{t}}
\end{gathered}
$$

The equations were solved at a given time step knowing the solution at the previous time step. Due to the parabolic nature of the boundary layer equations. the governing equations were solved at each time step by marching from station to station in the direction of the core flow. Solutions at a given station were obtained by solving the boundary layer equations sequentially. First. the momentum equation was solved for the velocity component in the core flow (streanwise) direction (ii). Second. continuity was solved for the velocity component in the $y$ direction (v). Then, the energy equation was solved for the temperature (T). If the station had not converged, the momentum, continuity, and energy equations were solved again for the velocity components and temperature. using the flow properties from the previous iteration. Convergence at a given station was obtained when the streamwise velocity components at all grid locations at that station converged. After the calculation at the station converged, the algorithm marched to the next streamwise station to solve for the boundary layer properties. This streamwise marching continued throughout the entire domain. Once the entire solution was obtained for the current time step. the boundary layer properties were used to calculate the core flow solution at the next time step.

Grid spacing perpendicular to the wall (y-direction) was based on an exponential function. The grid spacing was fine near the wall to better resolve the gradients at the wall. The grid spacing was course away from the wall where fine resolution of the gradients normal to the 
core stream were not necessary.

The derivative of the velocity at the wall (used to calculate wall shear stress) was determined using a second order approximation. Values at $\mathrm{j}=1$ (wall). $\mathrm{j}=2$. and $\mathrm{j}=\mathbf{3}$ were used.

$$
\left(\frac{\partial u}{\partial y}\right)_{i, 1}=\frac{y_{i, 3}^{2}\left(u_{t, 2}-u_{t, 1}\right)}{y_{i, 2} y_{i, 3}\left(y_{i, 3}-y_{t, 2}\right)}-\frac{y_{i, 2}^{2}\left(u_{i, 3}-u_{t, 1}\right)}{y_{t, 2} y_{i, 3}\left(y_{t, 3}-y_{i, 2}\right)}(15)
$$

The above algorithm worked well for flows with weak viscous/inviscid interaction, since the downstream influence could be neglected. However, when the flows were strongly interacting. such as those with strongly adverse pressure gradients or separation. downstream conditions had to be considered. The downstream influences were treated in two ways (I) the pressure gradient was differenced as a weighted average of forward and backward differences, and (2) a global pressure sweep was used. Both of these techuiques were based on the method of Davis and Barnett[2].

Specifically the pressure gradient was differenced as

$$
\frac{\partial \bar{P}}{\partial x}=(\epsilon)\left(\frac{\overline{P_{i j}}-\overline{P_{i-1 j}}}{x_{i}-x_{i-1}}\right)+(1-\epsilon)\left(\frac{\overline{P_{i+1 j}}-\overline{P_{i j}}}{x_{i+1}-x_{i}}\right)(16)
$$

where $\varepsilon$ is a weighting parameter of the forward and backward differencing. This term was required to remove the ellipticity in the PNS equations in strongly interacting flows. The quantity $\varepsilon$ determines what fraction of the forward difference of the pressure gradient that can be included so that the equations remain nonelliptic. If the flow was supersonic at a given $\mathrm{j}$ location, then only backward differencing was used for the pressure gradient $(\varepsilon=1)$. If the flow was subsonic then at a given $\mathrm{j}$ location then the following expression for $\varepsilon$ was used.

$$
\epsilon=\frac{\gamma M^{2}}{1+(\gamma-1) M^{2}}
$$

Most boundary layer methods use an inverse technique when the flow is separated. This requires a lot of computer time because the core flow and boundary layer regions must be modeled simultaneously. A method to convey the downstream influences upstream. while still maintaining the streamwise marching technique was considered. This made the algorithn much faster than other separated flow algorithms. Davis and Barnett[2] had modified the pressure terms in the supersonic para- bolized Navier-Stokes (PNS) equations to convey upstream influences. They had modified the pressure gradient terms using the downstream pressure. Because. the downstream pressure had been used, a global pressure sweep had been needed throughout the subsonic regions of the solution domain.

This pressure sweep technique also worked well with the uncoupled. unsteady PNS equations described above. The fictitious unsteady term that had been used by Davis and Barnett was not necessary in our case since the flow being modeled was. itself. unsteady. The equation for the pressure sweep was then

$$
P_{i j}=P_{i+1 j}-P_{i+1 j}^{\text {old }}+P_{i j}^{\text {old }}
$$

This pressure technique permitted the modeling of moderately separated flows. Downstream effects could be quickly propagated upstream. This method was made even faster by applying the pressure sweep only from a region of weak interaction (downstream of reattachment) to just upstream of the point of separation.

In addition. flaring of the advective term parallel to the core flow was used when the flow was separated. This was handled by taking the absolute value of the coefficient in front of the derivative of the advective term (RUB) and multiplying it by .1 to make it smaller. Again this operation was only performed when the flow was separated.

A modified Baldwin-Lomax model was used to account for turbulence. This model was a zero equation. eddy viscosity model. This model was faster than other turbulence models, such as the $k-\varepsilon$ two equation model. A modified model of Visbal and Knight was used to handle the effects of separation. Also to account for upstream turbulence history effects, a relaxation method of Shang and Hankey was employed. A detailed description of the turbulence model used is given in Sakowski. et. al [3].

This uncoupled boundary layer schenne worked well when used with the core flow solution already known. With this method. we can quickly determine the effects of boundary layers within inlets of supersonic jet engines. Thus, we can model several different geometric configurations and flow conditions in a reasonable amount of time.

\section{RESULTS AND DISCUSSION}

This uncoupled technique was compared with a tech- 
nique in which the boundary layer equations were solved simultaneously (coupled). The boundary layer equations and interactions with the inviscid core flow were set up the same way for both techniques. Each technique was executed until it converged to the same degree of accuracy. The uncoupled technique converged quickly, in 2 or 3 iterations. at a each streamwise station. The uncoupled technique tended to be 2.4 to 4.0 times faster than the coupled technique.

For the rest of this section we will attempt to verify the boundary layer algorithm and to judge the relative importance of various aspects of the algorithm. To do this we will look at two examples. The first example involves a case where the core flow is prescribed and the boundary layer is not interactive with the core flow. Specifically, the boundary layer algorithm will be used to model the flow conditions measured in experiments by Lewis. et al.[4]. For this example the core flow Mach number distribution was prescribed and was not interactive with the boundary layer code. The core flow Mach number distribution is shown on figure $2 \mathrm{~b}$. Calculations were started using a zero pressure gradient profile with a Reynolds number. based on momentum thickness, of about 4800 at $x=11.5$ inches downstream of the leading edge.

A second example involved a model of an actual supersonic inlet. In this example the boundary layer algorithm was used with the Large Perturbation Inlet code (LAPIN). LAPIN was used to calculate the core flow properties upstream and within a supersonic inlet. In this case the boundary layer code and LAPIN were interactive. Figure 1 shows the geometry of the NASA - LeRC 40-60 inlet. A free stream Mach number of 2.5 was used. The predictions of the combined boundary layer/core flow code were compared with the experimental results of Cubbison, et al.

\section{Dependence on grid spacing}

The first example. with the prescribed, non-interactive core flow. was nodeled using various numbers of grid points perpendicular to the core flow. If the number of grid points perpendicular to the core flow direction was too small (less than 20 in our case), the algorithm was unstable. Increasing the number of grid points made the algorithm stable. Good accuracy. however, is not obtained until an optimum number of grid points are used. When the number of grid points is too low the algorithm predicts friction coefficients that are lower than experimental results. To a point the predicted friction coefficient increased as the number of grid points was increased. However, the friction coefficient does not increase monotonically with the number of grid points. Beyond a certain number of grid points. the friction coefficient actually goes down as the number of grid points was increased. The point with the highest friction coefficient also seems to be closest to the experimental observations. This is the optinal number of grid points.

Figure $2 a$ shows the predicted friction coefficient versus $x$ for various numbers of grid points perpendicular to the core flow. In addition, the predicted results were compared with experimental results of Lewis, et al. [4] and the analytical model of Cebeci and Smith[6]. In this case. if the number of grid points was less than 100. the friction coefficient increased by increasing the grid points. Beyond 100 grid points the friction coefficient dropped by increasing the number of grid points. 100 grid points was the optimum number in terms of the accuracy of the results. However. any number of grid points between 50 and 100 yielded reasonable results. Obviously, increasing the number of grid points from 50 to 100 resulted in much longer computing times. The best number of grid points to use depends on the degree of accuracy that is required and the computing time that can be allocated. For instance, if one were interested in detailed wall effects, heat transfer or skin friction, it may be worth while to use 100 grid points. The accuracy is also dependent upon parameters used in the turbulence model. The results illustrated in figure $2 \mathrm{a}$ are for a given set of turbulent parameters used in the BaldwinLomax model. Sakowski, et al.[3] showed that for this particular case. increasing the number of grid points perpendicular to the wall required some adjustment 10 the turbulent parameters to yield results which more closely matched the experimental data.

However, if large scale flow properties are of concern. core flow Mach number and pressure, 50 points or less may be used. This was evident from the resuits of the second example. Figure 3 shows the dependency of the number of grid points perpendicular to the wall on the predicted core flow in the NASA - LeRC 40-60 Inlet. Note there are two boundary layers in this case. one on the centerbody and one on the cowl. The number of grid points refers to each boundary layer. not the total for both. As in the first example, when the number of grid points is too low ( 20 or less) the algorithm is unstable. In this case. the core flow and boundary layer codes were interactive, so the boundary layer results affected the core flow properties. For instance, when the number of grid points was increased to around 30 the algorithm was stable but the shock wave was predicted downstrean of the experimental observation. The predicted pressures away from the shock were reason- 
able even when only 30 grid points were used. When the number of grid points was increased to 40 the predicted shock wave position was further upstream and matched the experimental results better. When the number of grid points was increased beyond 40 the change in the core flow pressure was negligible. Thus. in this case there was no need to use more than 40 grid points perpendicular to the core flow.

\section{Dependence on Convergence criterion}

As described above the algorithm iterated until the velocities parallel to the core flow (u) converged. The convergence criterion at a given $x$ location was a fraction of the local core flow velocity $\left(u_{e}\right)$. Obviously. tightening the convergence criterion increased the computing time as well as increased the accuracy. It was found that if the convergence criterion was too loose. the solution was simply chaotic and unrealistic. Altering the convergence criterion had the biggest impact on the wall values. such as heat transfer. skin friction predictions and momentum thickness. As long as the convergence criterion was strict enough. the predicted flow properties were negligibly affected by the grid spacing in the $\mathrm{x}$ direction. And. if the core flow properties were of concern. the convergence criterion itself had only a small effect. In fact the convergence criterion can be as high as $10 \%$ of the local core flow velocity with only slight impact on the core flow properties. Figure 4 shows the predicted pressure as a function of position for flow in the 40-60 inlet for various levels of convergence. As shown on figure 4 the convergence criterion only affected the predicted core flow pressure near the throat of the inlet.

\section{Effect of upstream propagation}

In prescribing an inviscid core flow solution, the backward pressure sweep was not very significant in strongly interacting regions. i.e. shocks and adverse pressure gradients. The more significant mode of propagating effects upstream is the calculation of the pressure gradient as described in equation 16 . The forward differencing was necessary in adjusting the pressure gradient to provide a path for upstream propagation when the flow is subsonic. However. only a portion of the forward difference had be used so that the equations did not become elliptic. When the weighted forward/backward differencing of the pressure gradient was omitted, the results deviated from the experimental results.

However, it was found that the results were the same if pressure sweep was included or omitted. This is most likely due to the imposed core flow solution which already included downstream effects when the core flow was subsonic.

For the model of the 40-60 inlet. the pressure sweep had no influence on the predicted results. However the forward differencing of the pressure gradient had an effect. Figure 5 shows the predicted pressure distribution in the 40-60 inlet using the pressure gradient described in equations 16 and 17 and the predicted pressure distribution when only backward differencing of the pressure gradient was used $(\varepsilon=1)$. As expected. there was almost no difference in two results when the core flow was supersonic. since $\varepsilon=1$ was used in supersonic regions of the boundary layer in both cases. However. when the core flow was subsonic the predictions diverged from the experimental observation when only backward differencing was used.

\section{CONCLUSIONS}

Using the uncoupled boundary layer scheme was 2.4 to 4.0 times faster than a boundary layer scheme that solves the governing equations simultaneously. Thus. this method was a quick way to model boundary laves effects when a core flow algorithm was available.

Significant considerations were the grid spacing in the direction perpendicular to the wall. the differencing of the pressure gradient, and the convergence criterion. Changing the grid spacing and the convergence criterion had the largest impact when specific boundary layer properties were of concern. such and friction coefficient and nomentum thickness. The Baldwin-Lomax turbulence model. which was used to model turbulence. was shown by Sakowski. et al. [3] to be sensitive to the grid spacing. which in turn greatly affected the skin friction coefficient. If the convergence criteria was not small enough. the boundary layer algorithm was not robust in predicting boundary layer properties such as skin friction and momentum thickness. These parameters became highly sensitive to the grid spacing in the streamwise direction when the convergence criteria was not small enough.

However the grid spacing and convergence criterion had less of an impact on the overall core flow properties than the boundary layer properties. In fact. as long as the algorithm was stable. the number of grid points and convergence criterion could have many different values without having a significant effect on the core flow. The differencing of the pressure gradient seemed to have the largest impact on the core flow solution. The 
weighted average of forward and backward differencing resulted in predictions closer to the experimental observation than when only backward differencing was used. Finally, the changes made to the Baldwin-Lomax Turbulence model, described by Sakowski. et al.[3]. were significant to the accuracy of the results.

\section{REFERENCES}

1. O.K. Kwon, R.H. Pletcher, R.A. Delaney, "Solution Procedure for Unsteady Two-Dimensional Boundary Layer." ASME Journal of Fluids Engineering, Vol. 110, March 1988. pp 69-75.

2. R.T. Davis, M. Barnett, "The Calculation of Supersonic Viscous Flows using the Parabolized Navier-Stokes Equations." Computers and Fluids. Vol. 14. No. 3, pp 197-224, 1986.

3. B.A. Sakowski, D.D. Darling. R.L. Roach. A.G. van de Wall. "Evaluation and Application of the Baldwin-Lomax Turbulence Model in Two-Dimensional. Unsteady. Compressible Boundary Layers with and without Separation in Engine Inlets," AlAA 92-3676, 1992.

4. J.E. Lewis, R.L. Gran, T. Kubota. "An experiment on the Adiabatic Compressible Turbulent Boundary Layer in Adverse and Favorable Pressure Gradients," J. Fluid Mechanics 1972, Vol. 51, Part 4, pp 651-672.

5. R.W. Cubbison. E.T. Meleason, D.F.Johnson. "Performance Characteristics from Mach 2.58 to 1.98 of an Axisymmetric Mixed-Compression Inlet System with 60-Percent Internal Contraction." NASA TM X-1739. 1969.

6. T. Cebeci, A.M.O. Smith. "Analysis of Turbulent Boundary Layers." Academic Press, New York. 1974

\section{FIGURES}

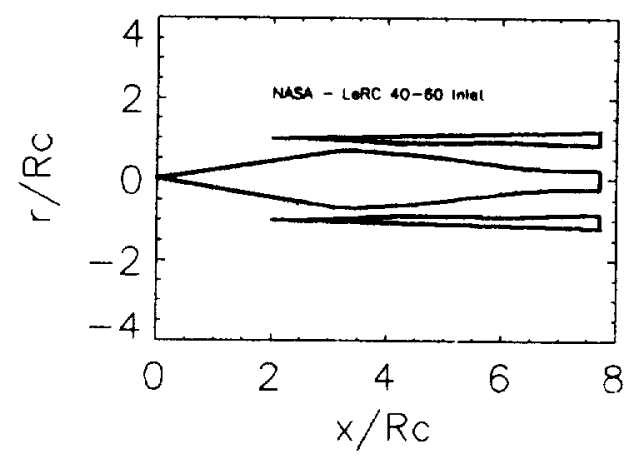

Figure 1 Geometry of NASA-LeRC 40-60 supersonic inlet.

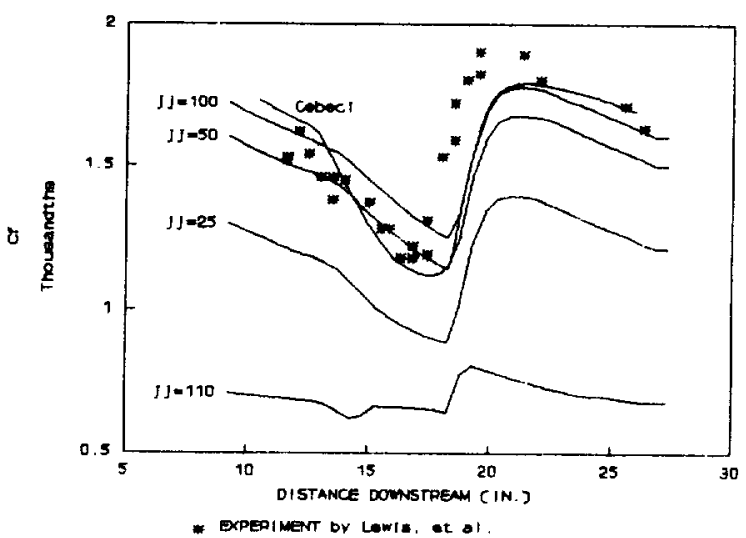

Figure 2a Friction Coefficient vs. Distance Downstream varying number of grid points perpendicular to wall.

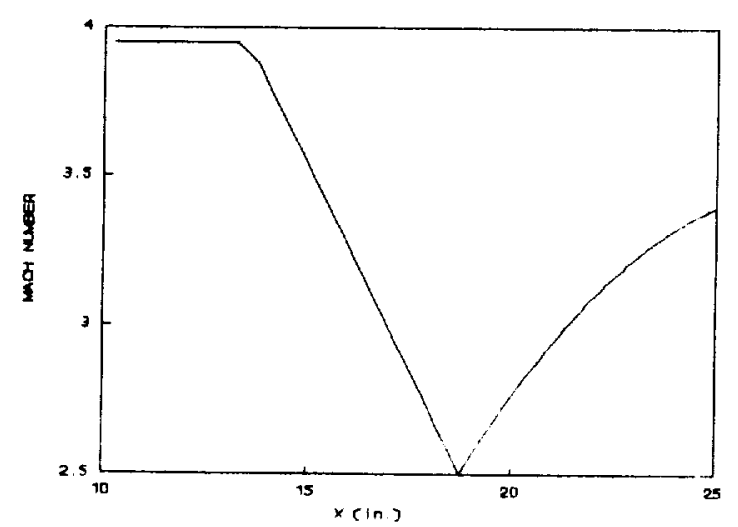

Figure 2b
Mach number vs. Position downstream - from experiment by Lewis. et al 


\section{FIGURES (con't)}

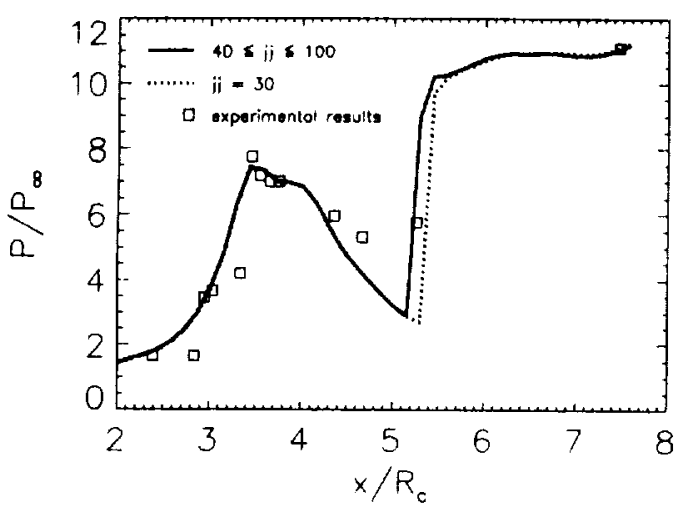

Figure 3 Predictions of pressure distribution for NASA-LeRC 40-60 inlet varying grid points perpendicular to wall.

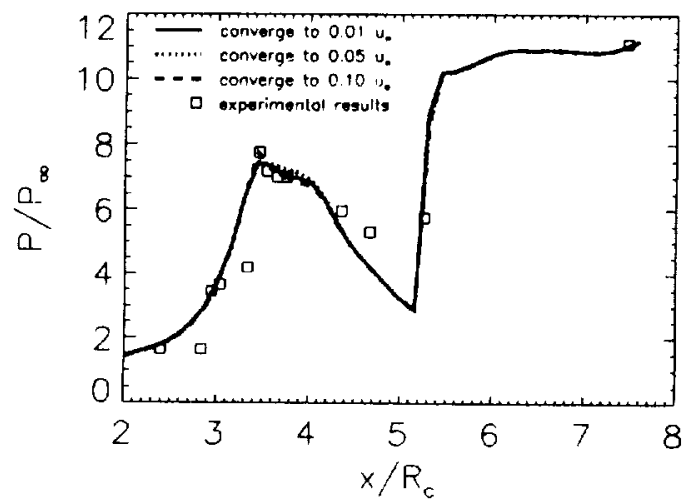

Figure 4 Predictions of pressure distributions of the NASA-LeRC 40-60 inlet varying the level of convergence.

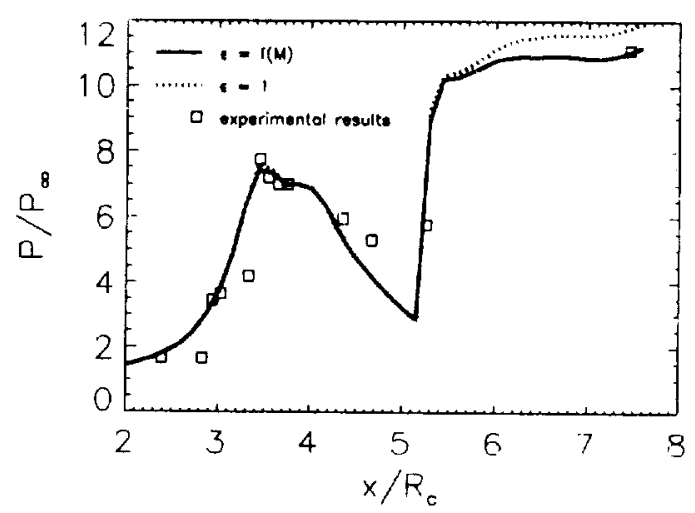

Figure 5

Predictions of pressure distribution in the NASA-LeRC 40-60 inlet varying the pressure gradient parameter $(E)$. 


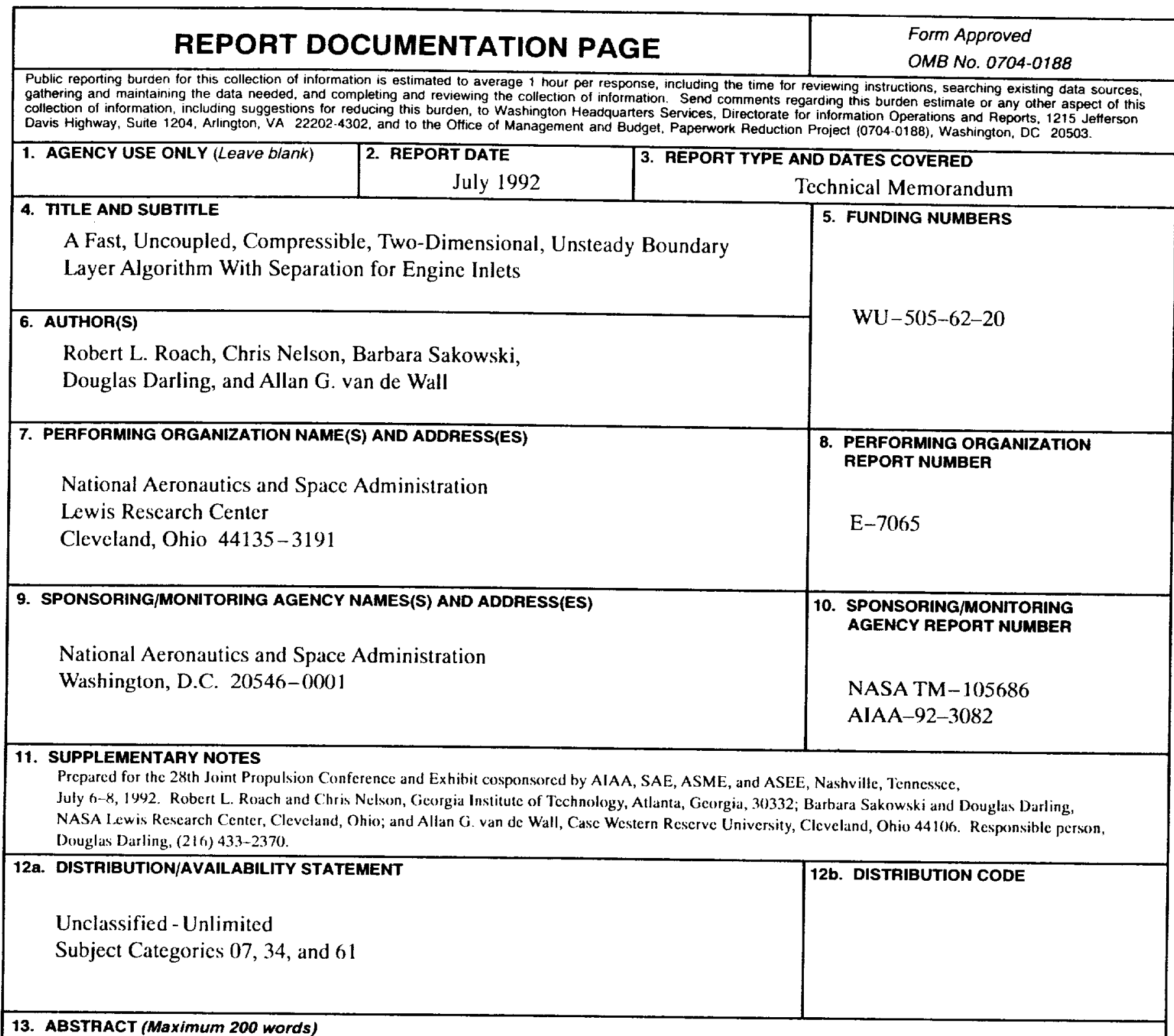

A finite difference boundary layer algorithm was developed to model viscous effects when an inviscid core flow solution is given. This algorithm solved each boundary layer equation separately, then iterated to find a solution. Solving the boundary layer equations sequentially, in this manner, was 2.4 to 4.0 times faster than solving the boundary layer equations simultaneously. This algorithm used a modified Baldwin-Lomax turbulence model, a weighted average of forward and backward differencing of the pressure gradient, and a backward sweep of the pressure. With these modifications, the boundary layer algorithm was able to model flows with and without separation. The number of grid points used in the boundary layer algorithm affected the stability of the algorithm as well as the accuracy of the predictions of friction coefficients and momentum thicknesses. Results of this boundary layer algorithm compared well with experimental observations of friction coefficients and momentum thicknesses. In addition, when used interactively with an inviscid flow algorithm, this boundary layer algorithm corrected for viscous effects to give a good match with experimental observations for pressures in a supersonic inlet.

\section{SUBJECT TERMS}

Boundary layer flow; Numerical analysis compressible flow; Intake systems

17. SECURITY CLASSIFICATION
OF REPORT
Unclassified

Unclassified

18. SECUAITY CLASSIFICATION
OF THIS PAGE
Unclassified

Unclassified
19. SECURTY CLASSIFICATION OF ABSTRACT

20. LIMITATION OF ABSTRACT 